\title{
AVALIAÇÃO DE SEIS PROTOCOLOS PRÉ-ANESTÉSICOS PARA ANESTESIA EPIDURAL DE CANINOS*
}

\author{
EVALUATION OF SIX PRE-ANESTHETIC COMBINATIONS TO CANINE \\ EPIDURAL ANESTHESIA
}

\section{SILVIAPRAXEDES DO CANTO $^{1}$ \& JOÃO ROBERTO BRAGA DE MELLO ${ }^{2,3}$}

\begin{abstract}
RESUMO
Vários protocolos de pré-anestesia podem ser usados em esquemas de anestesia que incluam anestesia epidural. A disponibilidade de diferentes fármacos e combinações permite a escolha de um procedimento anestésico específico para cada paciente, dependendo do grau de risco anestésico e do temperamento do animal. A escolha do protocolo pré anestésico deve ser criteriosa, permitindo a realização correta da técnica de anestesia epidural, sem abrir mão de critérios de segurança para o paciente. 0s objetivos do trabalho foram avaliar e comparar os efeitos de seis protocolos pré-anestésicos sobre diversas funções orgânicas, em esquemas de anestesia que incluem a epidural. As funções cardiocirculatória e respiratória foram avaliadas por intermédio de eletrocardiograma, determinação da pressão arterial sistólica e gasometria sangüínea. Os tempos de latência da medicação pré-anestésica, tempo até o aparecimento de relaxamento anal após a epidural e de latência da epidural foram determinados. Com relação à função cardiocirculatória, os protocolos Atropina + Clorpromazina + Buprenorfina (ACB) e Midazolam (M) produziram aumento na freqüência cardíaca após a realização da epidural, e Atropina + Xilazina (AX) foi responsável por marcada redução da freqüência cardíaca imediatamente após a medicação pré-anestésica (MPA). A pressão arterial sistólica foi elevada com o protocolo $\mathrm{M} \mathrm{e}$ reduzida com o protocolo $\mathrm{AX}$, cinco e dez minutos após a MPA, respectivamente. Diferentes alterações do ritmo cardíaco foram observadas, dependendo do protocolo utilizado, porém sem significância clínica. Todos os protocolos causaram aumentos na pressão parcial de $\mathrm{CO}_{2}$ no sangue venoso, variando nos diferentes tempos de análise. Nenhum causou sedação adequada para a realização da anestesia epidural, sendo recomendável suplementação da pré-anestesia.
\end{abstract}

Descritores: pré-anestésicos, epidural, cães, atropina, clorpromazina, buprenorfina, midazolam, xilazina.

\begin{abstract}
Various pre-anaesthetic protocols can be used in an anaesthetic regimen that includes epidural anesthesia. Different drug availability and drug combinations allows adjusting the anaesthetic procedure to each patient, concerning to the risk grade and temperament. The choosing of a pre-anaesthetic protocol needs to be judicious, to allow the correct execution of the epidural anaesthetic technique, without challenging its safety. The present study was undertaken to evaluate and compare the effect six distinct preanaesthetic protocols on different organic functions, in anaesthetic regimens that include the epidural anesthesia. Cardiocirculatory and respiratory functions were evaluated by means of electrocardiogram, determination of systolic blood pressure and blood gas analysis. The latency times of pre-anaesthetic medication, time to anal relaxation, and occurrence of epidural anesthesia were determined. Concerning the cardiocirculatory function it was evident that the protocols Atropine + Chlorpromazine + Buprenorphine (ACB) and Midazolan (M) caused an increase of the heart rate after the epidural, and Atropine + Xylazine protocol (AX) decreased the heart rate after the pre-anaesthetic medication. Significant decrease of the systolic arterial blood pressure was observed $10 \mathrm{~min}$ after pre-anaesthetic medication with $\mathrm{AX}$ and increase $5 \mathrm{~min}$ after pre-anaesthetic medication with $\mathrm{M}$ protocol. In spite of the occurrence of cardiac arrhythmia with all protocols, they were transitory and without clinical significance. All protocols caused increase of $\mathrm{CO}_{2}$ partial pressure after different periods of time. None of the protocols caused adequate sedation for the accomplishment of the epidural anesthesia and must be necessary a pre-anaesthetic supplementation.
\end{abstract}

Key words: pre-anaesthetic agents, epidural, dogs, atropine, chlorpromazine, buprenorphine, midazolan, xylazine. 


\section{INTRODUÇÃO}

A anestesia epidural é uma técnica anestésica consagrada, que tem como principais vantagens a segurança, eficiência e baixo custo [10,20]. A ausência quase total de depressão cárdio-respiratória favorece sua indicação como técnica anestésica alternativa para animais de alto risco, onde a anestesia geral estaria contra-indicada [1]. Paralelamente, a anestesiologia veterinária evoluiu de forma considerável nas últimas décadas, através de inúmeras pesquisas. Graças aos conhecimentos adquiridos, atualmente a anestesia geral pode ser efetuada de modo mais seguro e prontamente reversível. As técnicas de anestesia geral por infusão e o uso de antagonistas farmacologicamente específicos, bem como os conhecimentos cada vez mais sólidos de anestesia balanceada, são exemplos desta realidade [25]. Sendo assim, o que há vinte anos era considerado um protocolo anestésico seguro a um animal de alto risco, atualmente pode, e der ser questionado [5].

Em casos excepcionais, de pacientes de comportamento dócil e calmo, a técnica de anestesia epidural pode ser realizada sem tranqüilização prévia. Entretanto, na maior parte dos cães se faz necessária uma potente tranqüilização para que a técnica seja efetuada de forma correta [1, 18]. Nas situações em que o animal apresenta comportamento agressivo e alto grau de risco anestésico, uma conduta anestésica que inclua a anestesia epidural pode deixar de ser uma indicação para se tornar negligência, visto que o grau de tranqüilização exigido põe em questão a segurança anestésica [5]. Nesta situação, poderia ser mais adequado, uma anestesia geral moderada devidamente amparada na monitorização cuidadosa do paciente. Diante deste contexto, faz-se necessário o conhecimento mais detalhado do comportamento de diferentes protocolos préanestésicos especificamente para a anestesia epidural, a fim de que estes possam ser escolhidos de forma criteriosa, adequado-se assim a conduta anestésica ao paciente em questão.

A medicação pré-anestésica constitui parte de um protocolo anestésico que inclui a anestesia epidural. Inúmeros procedimentos pré-anestésicos podem ser utilizados em busca da tranqüilização exigida para a fácil execução da anestesia epidural e para um período de trans e pós-operatório sem intercorrências. Geralmente, a escolha se baseia em dois aspectos fundamentais: o temperamento do paciente e seu grau de risco $[1,10]$. Cães de alto risco exigem préanestésicos pouco depressores, enquanto que cães agressivos necessitam os muito depressores. Todavia o que determina a escolha de um protocolo préanestésico muito depressor é o temperamento agressivo do paciente, independente de seu grau de risco ser alto ou baixo [7].

Foram escolhidos para estes estudo fármacos integrantes dos principais grupos farmacológicos préanestésicos, de uso rotineiro em anestesiologia veterinária e suas combinações, por diferentes vias de administração. Estas vem sendo indicadas na préanestesia de uma forma geral, e não especificamente para a anestesia epidural.

Este trabalho objetivou avaliar 6 protocolos préanestésicos diante das principais funções orgânicas, especialmente em condutas anestésicas que incluem a anestesia epidural.

\section{MATERIAIS E MÉTODOS}

Foram usados 30 cães adultos, de ambos os sexos, sem raça definida, com peso médio de $8,9 \pm$ $3,2 \mathrm{~kg}$. Os animais foram submetidos a jejum alimentar de 12 horas e restrição hídrica de 2 horas antes do procedimento anestésico. Foram divididos aleatoriamente em 6 grupos experimentais de 5 animais:

$\mathrm{AX}=$ sulfato de atropina ${ }^{1}\left(0,044 \mathrm{mg} \cdot \mathrm{kg}^{-1} \mathrm{SC}\right)+$ cloridrato de xilazina ${ }^{2}\left(2 \mathrm{mg} \cdot \mathrm{kg}^{-1} \mathrm{IM}\right)$;

$\mathrm{ACB}=$ sulfato de atropina $\left(0,044 \mathrm{mg} \cdot \mathrm{kg}^{-1} \mathrm{SC}\right)+$ cloridrato de clorpromazina ${ }^{3}\left(1 \mathrm{mg} \cdot \mathrm{kg}^{-1} \mathrm{IV}\right)+$ cloridrato de buprenorfina ${ }^{4}\left(5 \mu \mathrm{g} \mathrm{kg}^{-1} \mathrm{IV}\right)$;

$\mathrm{MC}=$ midazolam $^{5}\left(0,3 \mathrm{mg} \cdot \mathrm{kg}^{-1} \mathrm{IV}\right)+$ cloridrato de clorpromazina ( $\left.1 \mathrm{mg} \cdot \mathrm{kg}^{-1} \mathrm{IV}\right)$;

$\mathrm{AMB}=$ sulfato de atropina $\left(0,044 \mathrm{mg} \cdot \mathrm{kg}^{-1} \mathrm{SC}\right)+$ midazolam $\left(0,5 \mathrm{mg} \cdot \mathrm{kg}^{-1} \mathrm{IM}\right)+$ cloridrato de buprenorfina (10 $\left.\mu \mathrm{g} \cdot \mathrm{kg}^{-1} \mathrm{IM}\right)$;

$\mathrm{C}=$ cloridrato de clorpromazina $\left(1 \mathrm{mg} \cdot \mathrm{kg}^{-1} \mathrm{IV}\right)$;

$\mathrm{M}=$ midazolam $\left(0,5 \mathrm{mg} \cdot \mathrm{kg}^{-1} \mathrm{IM}\right)$. 
A seqüência de procedimentos realizados nos diferentes grupos experimentais está resumido na Figura 1.

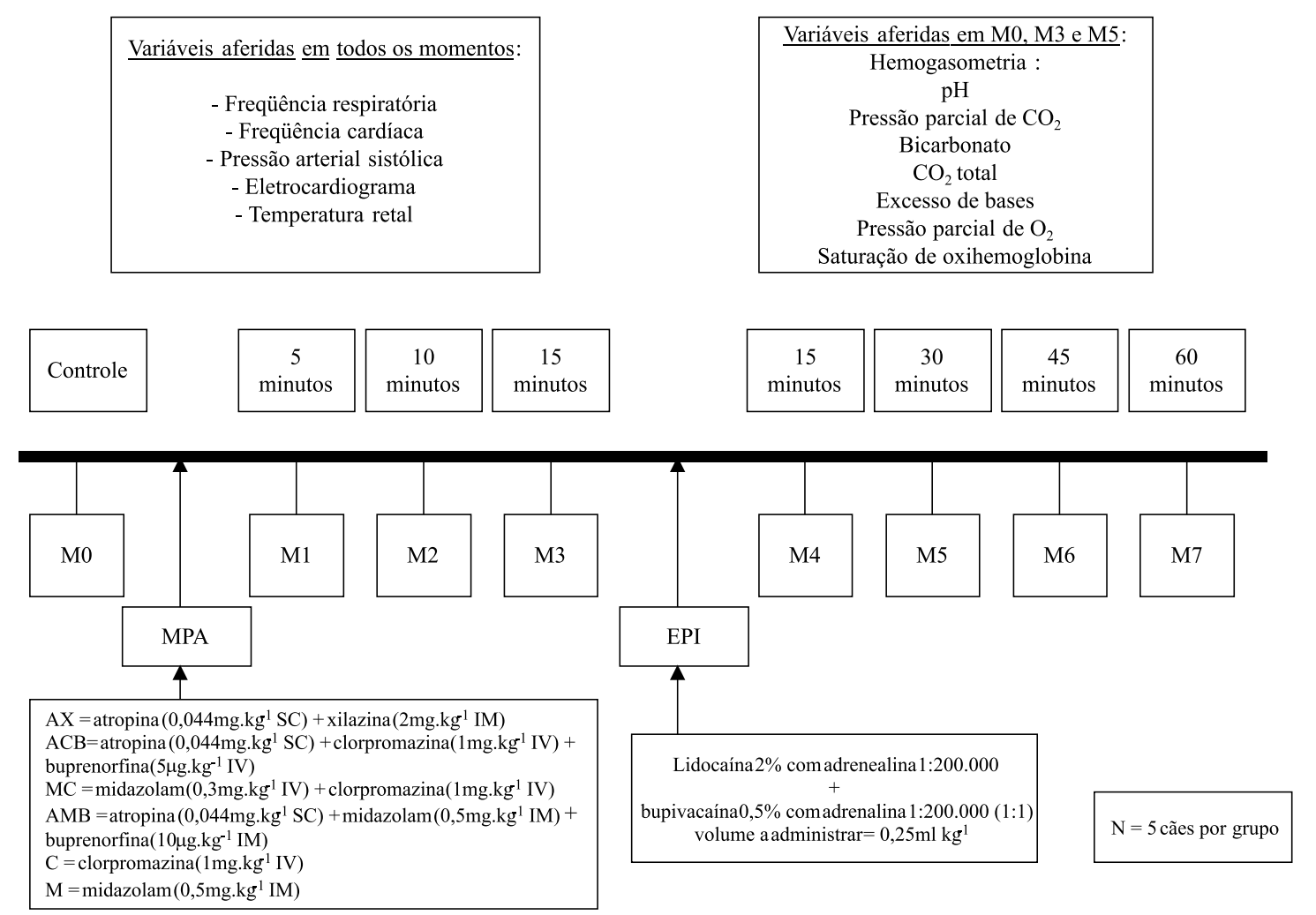

Figura 1. Esquema do desenvolvimento experimental.

A anestesia epidural foi realizada com lidocaína $2 \%$ com adrenalina $1: 200000^{6}$ e bupivacaína $0,5 \% \mathrm{com}$ adrenalina $1: 200000^{7}(1: 1)$, em volume de $0,25 \mathrm{ml} . \mathrm{kg}^{-1}$, 20 minutos após a MPA e obedeceu a técnica descrita por Skarda (1996). Concluída a anestesia epidural, os animais foram mantidos em decúbito esternal por mais 15 minutos, minimizando a difusão cranial da solução anestésica e possibilitando o bloqueio bilateral.

A freqüência respiratória foi estimada pela observação dos movimentos de expansão torácica e representada em movimentos por minuto. $\mathrm{O}$ eletrocardiograma foi registrado em aparelho portátil ${ }^{8}$, nas 6 derivações de Einthoven [4] na velocidade de 50 milímetros por segundo e sensibilidade de 1 milivolt, imediatamente antes da MPA (M0), para a realização do diagnóstico eletrocardiográfico e nos demais momentos na derivação II. Através do ECG foram medidas a freqüência cardíaca, amplitude e duração de onda $\mathrm{P}$, duração do intervalo PR e complexo QRS, amplitude da onda $R$, alterações da onda $T$ e do segmento ST e do ritmo cardíaco. A pressão arterial sistólica foi aferida através de aparelho de Doppler ${ }^{9}$, registrada em milímetros de mercúrio, conforme descrito anteriormente [9]. A temperatura retal foi aferida por meio de termômetro clínico digita ${ }^{10} \mathrm{e}$ registrada em graus centígrados [12].

A coleta de sangue venoso para hemogasometria foi realizada mediante a punção da veia jugular esquerda [3]. O sangue heparinizado e mantido em condições anaeróbicas foi enviado em resfriamento, no máximo em 2 horas, para análise no Laboratório de Hemo-gasometria e Análises Clínicas do Instituto de Cardiologia do Rio Grande do Sul, sendo processado em analisador de $\mathrm{pH}$ e gases sangüíneos digital automatizado $^{11}$. 
Foram avaliadas ainda a necessidade de suplementação de MPA, as latências da MPA e da anestesia epidural, registradas em minutos. $\mathrm{O}$ tempo em segundos até o estabelecimento de relaxamento anal após a epidural também foi registrado.

Os dados obtidos foram analisados pela análise da variância (ANOVA), com auxílio do programa SPSS. Foi realizado o teste de múltiplas comparações de Tukey, de acordo com Cox [6] para demonstrar diferenças entre os protocolos de MPA e entre os momentos de coleta. $\mathrm{P}<0,05$.

\section{RESULTADOS}

Os resultados de freqüência respiratória, freqüência cardíaca, pressão arterial sistólica e temperatura retal estão apresentados na tabela 1 . Houve redução da freqüência respiratória após a administração da MPA com todos os protocolos, exceto com o protocolo $\mathrm{M}$, onde foi observada elevação da freqüência respiratória. As únicas diferenças estatisticamente significativas ocorreram com o protocolo AX em M4 (15 minutos após a EPI) e M6 (45 minutos após a EPI).

Tabela 1. Freqüência respiratória em movimentos por minuto, freqüência cardíaca em batimentos por minuto, pressão arterial sistólica em milímetros de mercúrio e temperatura retal em graus centígrados (médias \pm desvio padrão) de cães ( $\mathrm{n}=5$ por grupo) submetidos a seis protocolos pré-anestésicos e então à epidural com lidocaína $2 \%$ com adrenalina 1:200000 + bupivacaína 0,5\% com adrenalina $1: 200000(1: 1)$.

\begin{tabular}{|c|c|c|c|c|c|c|c|c|c|}
\hline $\begin{array}{l}\text { Tempos } \\
\text { Variável }\end{array}$ & & Mo & M1 & M2 & M3 & M4 & M5 & M6 & M7 \\
\hline & $\begin{array}{l}\text { MPA } \\
\text { AX }\end{array}$ & $21,6 \pm 2,1^{2}$ & $15,2 \pm 7,1^{12 a b}$ & $14,4 \pm 9,6^{12 a}$ & $8,8 \pm 5,2^{12 a}$ & $6,4 \pm 3,5^{\text {la }}$ & $11,2 \pm 9,8^{12}$ & $7,2 \pm 5,2^{1 \mathrm{a}}$ & $8,0 \pm 4,0^{12 \mathrm{a}}$ \\
\hline & $\mathrm{ACB}$ & $20,0 \pm 8,0$ & $13,6 \pm 4,5^{\mathrm{a}}$ & $14,4 \pm 3,5^{\mathrm{a}}$ & $13,6 \pm 4,5^{\mathrm{a}}$ & $11,2 \pm 3,3^{\mathrm{ab}}$ & $12,0 \pm 4,0$ & $13,6 \pm 4,5^{\mathrm{ab}}$ & $12,8 \pm 3,3^{\mathrm{ab}}$ \\
\hline Freqüência & $\mathrm{MC}$ & $17,0 \pm 5,5$ & $24,0 \pm 14,1^{\mathrm{ab}}$ & $24,8 \pm 13,6^{\mathrm{ab}}$ & $17,6 \pm 6,6^{\mathrm{ab}}$ & $12,8 \pm 5,2^{\mathrm{ab}}$ & $12,8 \pm 3,3$ & $11,2 \pm 3,3^{\mathrm{ab}}$ & $13,6 \pm 4,5^{\mathrm{ab}}$ \\
\hline \multirow[t]{5}{*}{ Respiratória } & AMB & $24,0 \pm 14,1$ & $35,2 \pm 13,0^{\mathrm{b}}$ & $30,4 \pm 7,2^{\mathrm{ab}}$ & $36,0 \pm 9,8^{b}$ & $18,4 \pm 7,7^{\mathrm{bc}}$ & $18,8 \pm 10,1$ & $19,2 \pm 9,7^{\mathrm{ab}}$ & $18,4 \pm 6,6^{\mathrm{ab}}$ \\
\hline & $\mathrm{C}$ & $18,4 \pm 4,5$ & $16,8 \pm 10,3^{\mathrm{ab}}$ & $12,0 \pm 2,8^{\mathrm{a}}$ & $16,0 \pm 4,0^{\mathrm{ab}}$ & $10,0 \pm 2,0^{\mathrm{ab}}$ & $11,0 \pm 2,4$ & $12,8 \pm 4,3^{\mathrm{ab}}$ & $10,4 \pm 4,5^{\mathrm{a}}$ \\
\hline & M & $19,6 \pm 7,7$ & $31,2 \pm 12,1^{\mathrm{ab}}$ & $38,4 \pm 18,0^{b}$ & $30,4 \pm 9,8^{\mathrm{ab}}$ & $24,8 \pm 9,5^{\mathrm{c}}$ & $23,2 \pm 10,7$ & $20,8 \pm 5,9^{\mathrm{b}}$ & $23,2 \pm 9,1^{b}$ \\
\hline & $\mathrm{AX}$ & $117 \pm 28,8^{12}$ & $66 \pm 17,1^{12 a}$ & $60 \pm 28^{1 \mathrm{a}}$ & $114 \pm 86^{12}$ & $138 \pm 35,8^{2}$ & $114 \pm 35^{12}$ & $105 \pm 9^{12 \mathrm{ab}}$ & $105 \pm 8^{12 a b}$ \\
\hline & $\mathrm{ACB}$ & $129 \pm 22,7$ & $114 \pm 22,7^{\mathrm{ab}}$ & $90 \pm 18,3^{\mathrm{ab}}$ & $141 \pm 63,2$ & $159 \pm 57,7$ & $177 \pm 63,1$ & $165 \pm 49^{\mathrm{ab}}$ & $159 \pm 43^{\mathrm{ab}}$ \\
\hline Freqüência & $\mathrm{MC}$ & $150 \pm 15,0$ & $138 \pm 16,4^{b}$ & $135 \pm 2,1^{\text {bc }}$ & $126 \pm 17,1$ & $120 \pm 35,1$ & $126 \pm 31,1$ & $153 \pm 29^{\mathrm{ab}}$ & $156 \pm 8,2^{\mathrm{ab}}$ \\
\hline \multirow{5}{*}{ Cardíaca } & AMB & $138 \pm 32,5$ & $129 \pm 36,1^{b}$ & $132 \pm 37,3^{\mathrm{bc}}$ & $165 \pm 35,1$ & $147 \pm 32,5$ & $132 \pm 37,3$ & $138 \pm 20^{\mathrm{ab}}$ & $138 \pm 19^{\mathrm{ab}}$ \\
\hline & $\mathrm{C}$ & $135 \pm 15,0$ & $108 \pm 35,8^{\mathrm{ab}}$ & $102 \pm 26,8^{\mathrm{bc}}$ & $96 \pm 22,7$ & $87 \pm 28,8$ & $105 \pm 43,7$ & $99 \pm 36,1^{\mathrm{a}}$ & $99 \pm 37,6^{\mathrm{a}}$ \\
\hline & M & $138 \pm 26,8$ & $138 \pm 12,5^{b}$ & $150 \pm 15^{c}$ & $153 \pm 19,5$ & $162 \pm 50,1$ & $162 \pm 50,1$ & $171 \pm 46,9^{b}$ & $168 \pm 46,7^{b}$ \\
\hline & $\mathrm{AX}$ & $177 \pm 15,2^{\mathrm{ab}}$ & $170 \pm 29,7$ & $139 \pm 27,0^{1}$ & $178 \pm 38,3$ & $197 \pm 13,0$ & $182 \pm 48,8$ & $162 \pm 33,6$ & $174 \pm 43,7$ \\
\hline & $\mathrm{ACB}$ & $131 \pm 53,6^{\mathrm{a}}$ & $160 \pm 77,4$ & $148 \pm 64,9$ & $127 \pm 65,3$ & $149 \pm 72,0$ & $160 \pm 68,8$ & $141 \pm 69,3$ & $145 \pm 63,8$ \\
\hline Pressão & $\mathrm{MC}$ & $236 \pm 37,8^{b}$ & $211 \pm 68,9$ & $209 \pm 64,2$ & $199 \pm 61,2$ & $182 \pm 50,6$ & $181 \pm 52,0$ & $174 \pm 54,1$ & $170 \pm 52,4$ \\
\hline Arterial & AMB & $199 \pm 47,7^{\mathrm{ab}}$ & $175 \pm 75,1$ & $174 \pm 82,6$ & $172 \pm 72,5$ & $245 \pm 69,2$ & $236 \pm 55.9$ & $232 \pm 87,0$ & $228 \pm 96,2$ \\
\hline \multirow[t]{4}{*}{ Sistólica } & $\mathrm{C}$ & $193 \pm 82,1^{\text {ab }}$ & $182 \pm 76,6$ & $185 \pm 83,3$ & $190 \pm 76,8$ & $200 \pm 45,4$ & $200 \pm 51,8$ & $193 \pm 47,1$ & $183 \pm 63,4$ \\
\hline & M & $141 \pm 29,2^{\mathrm{ab}}$ & $204 \pm 90,7$ & $226 \pm 66,5$ & $220 \pm 51,4$ & $216 \pm 78,9$ & $208 \pm 77,1$ & $212 \pm 80,9$ & $226 \pm 79,2$ \\
\hline & $\mathrm{AX}$ & $38,5 \pm 0,2^{2}$ & $38,5 \pm 0,4^{2}$ & $38,3 \pm 0,5^{2}$ & $38,2 \pm 0,4^{2}$ & $36,7 \pm 1,4^{12}$ & $35,9 \pm 1,3^{1}$ & $35,3 \pm 1,5^{1}$ & $34,8 \pm 1,5^{1}$ \\
\hline & $\mathrm{ACB}$ & $38,9 \pm 0,3^{2}$ & $38,7 \pm 0,5^{2}$ & $38,4 \pm 0,5^{2}$ & $38,0 \pm 0,4^{2}$ & $36,3 \pm 0,4^{1}$ & $35,6 \pm 0,7^{1}$ & $35,3 \pm 1,0^{1}$ & $35,2 \pm 1,2^{1}$ \\
\hline Temperatura & $\mathrm{MC}$ & $38,9 \pm 0,4^{3}$ & $38,4 \pm 0,3^{3}$ & $38,2 \pm 0,3^{3}$ & $38,0 \pm 0,3^{3}$ & $35,9 \pm 0,5^{2}$ & $35,3 \pm 0,6^{12}$ & $34,9 \pm 0,8^{12}$ & $34,7 \pm 0,9^{1}$ \\
\hline \multirow[t]{3}{*}{ Retal } & $\mathrm{AMB}$ & $39,2 \pm 0,4^{3}$ & $39,0 \pm 0,3^{3}$ & $38,8 \pm 0,2^{3}$ & $38,6 \pm 0,2^{3}$ & $37,0 \pm 0,6^{2}$ & $36,3 \pm 0,3^{12}$ & $36,0 \pm 0,5^{1}$ & $35,9 \pm 0,4^{1}$ \\
\hline & $\mathrm{C}$ & $39,1 \pm 0,3^{2}$ & $38,9 \pm 0,2^{2}$ & $38,7 \pm 0,2^{2}$ & $38,6 \pm 0,2^{2}$ & $36,9 \pm 0,9^{1}$ & $36,3 \pm 0,9^{1}$ & $35,8 \pm 1,4^{1}$ & $35,8 \pm 1,5^{1}$ \\
\hline & $\mathrm{M}$ & $38,8 \pm 0,4^{2}$ & $38,7 \pm 0,2^{2}$ & $38,6 \pm 0,3^{2}$ & $38,3 \pm 0,5^{2}$ & $36,6 \pm 0,8^{1}$ & $35,9 \pm 0,6^{1}$ & $36,1 \pm 0,7^{1}$ & $36,2 \pm 0,5^{1}$ \\
\hline
\end{tabular}

Para cada variável, letras diferentes na mesma coluna representam diferença estatisticamente significativa entre os protocolos, e números diferentes na mesma linha representam diferença estatisticamente significativa entre os momentos $(\mathrm{p}<0,05)$.

$\mathrm{AX}=$ atropina + xilazina; $\mathrm{ACB}=$ atropina + clorpromazina + buprenorfina; $\mathrm{MC}=$ midazolam + clorpromazina; $\mathrm{AMB}=$ atropina + midazolam + buprenorfina; $\mathrm{C}=$ clorpromazina; $\mathrm{M}=$ midazolam. $\mathrm{MPA}=$ medicação pré-anestésica; $\mathrm{EPI}=$ epidural; $\mathrm{M} 0=$ controle; M1 = 5 minutos após a MPA; M2 = 10 minutos após a MPA; M3 = 15 minutos após a MPA; M4 = 15 minutos após a EPI; M5 = 30 minutos após a EPI; M6 $=45$ minutos após a EPI; M7 $=60$ minutos após a EPI. 
Tabela 2. pH, bicarbonato e excesso de bases sangüíneos (médias \pm desvio padrão) de cães ( $\mathrm{n}=5$ por grupo) submetidos a seis protocolos pré-anestésicos e então à epidural com lidocaína $2 \%$ com adrenalina 1:200000 + bupivacaína $0,5 \%$ com adrenalina $1: 200000(1: 1)$.

\begin{tabular}{|c|c|c|c|c|}
\hline $\begin{array}{l}\text { Tempos } \\
\text { Variável } \\
\end{array}$ & MPA & M0 & M3 & M5 \\
\hline \multirow{6}{*}{$\mathrm{pH}$} & $\mathrm{AX}$ & $7,25 \pm 0,07^{\mathrm{a}}$ & $7,22 \pm 0,10^{\mathrm{a}}$ & $7,21 \pm 0,11^{\mathrm{a}}$ \\
\hline & $\mathrm{ACB}$ & $7,34 \pm 0,04^{\mathrm{bc}}$ & $7,38 \pm 0,01^{\mathrm{b}}$ & $7,32 \pm 0,05^{\mathrm{ab}}$ \\
\hline & $\mathrm{MC}$ & $7,28 \pm 0,03^{\mathrm{ab}}$ & $7,29 \pm 0,03^{\mathrm{ab}}$ & $7,26 \pm 0,05^{\mathrm{ab}}$ \\
\hline & AMB & $7,38 \pm 0,01^{2 c}$ & $7,37 \pm 0,01^{2 b}$ & $7,32 \pm 0,03^{\text {lab }}$ \\
\hline & $\mathrm{C}$ & $7,38 \pm 0,03^{c}$ & $7,36 \pm 0,03^{b}$ & $7,35 \pm 0,05^{\mathrm{b}}$ \\
\hline & M & $7,37 \pm 0,03^{\mathrm{bc}}$ & $7,35 \pm 0,02^{b}$ & $7,31 \pm 0,03^{\mathrm{ab}}$ \\
\hline \multirow{7}{*}{$\begin{array}{l}\text { Bicarbonato } \\
\text { Sangüíneo } \\
(\mathrm{mEq} / \mathrm{l})\end{array}$} & $\mathrm{AX}$ & $22,4 \pm 3,0^{\mathrm{a}}$ & $22,4 \pm 2,9^{a}$ & $21,6 \pm 3,5^{\mathrm{a}}$ \\
\hline & ACB & $26,4 \pm 2,6^{\mathrm{ab}}$ & $27,2 \pm 2,0^{b}$ & $27,0 \pm 1,8^{\mathrm{bc}}$ \\
\hline & $\mathrm{MC}$ & $22,4 \pm 2,8^{\mathrm{a}}$ & $21,2 \pm 1,9^{\mathrm{a}}$ & $22,0 \pm 2,7^{\mathrm{ab}}$ \\
\hline & AMB & $28,0 \pm 1,5^{b}$ & $28,0 \pm 1,2^{\mathrm{b}}$ & $28,2 \pm 1,7^{\mathrm{c}}$ \\
\hline & $\mathrm{C}$ & $28,4 \pm 2,1^{b}$ & $28,6 \pm 1,6^{b}$ & $28,2 \pm 2,4^{\mathrm{c}}$ \\
\hline & M & $26,0 \pm 2,7^{\mathrm{ab}}$ & $26,8 \pm 2,2^{b}$ & $26,4 \pm 2,8^{\mathrm{abc}}$ \\
\hline & $\mathrm{AX}$ & $-4,6 \pm 4,3^{\mathrm{a}}$ & $-5,2 \pm 4,6^{\mathrm{a}}$ & $-6,4 \pm 5,0^{\mathrm{a}}$ \\
\hline \multirow{5}{*}{$\begin{array}{l}\text { Excesso de } \\
\text { Bases } \\
(\mathrm{mEq} / \mathrm{l})\end{array}$} & ACB & $1,2 \pm 2,3^{\mathrm{bc}}$ & $1,8 \pm 2,1^{\mathrm{b}}$ & $0,8 \pm 2,3^{\mathrm{bc}}$ \\
\hline & $\mathrm{MC}$ & $-4,0 \pm 3,0^{\mathrm{ab}}$ & $-5,0 \pm 2,4^{a}$ & $-4,8 \pm 2,7^{\mathrm{ab}}$ \\
\hline & AMB & $3,0 \pm 1,5^{\mathrm{c}}$ & $2,6 \pm 1,1^{b}$ & $2,0 \pm 2,0^{c}$ \\
\hline & $\mathrm{C}$ & $3,6 \pm 1,6^{c}$ & $3,0 \pm 2,4^{b}$ & $3,0 \pm 3,3^{\mathrm{c}}$ \\
\hline & M & $0,1 \pm 2,5^{\mathrm{abc}}$ & $1,2 \pm 2,5^{b}$ & $0,1 \pm 3,2^{\mathrm{abc}}$ \\
\hline
\end{tabular}

Para cada variável, letras diferentes na mesma coluna representam diferença estatisticamente significativa entre os protocolos, e números diferentes na mesma linha representam diferença estatisticamente significativa entre os momentos $(\mathrm{p}<0,05)$.

$\mathrm{AX}=$ atropina + xilazina; $\mathrm{ACB}=$ atropina + clorpromazina + buprenorfina; $\mathrm{MC}=$ midazolam + clorpromazina; $\mathrm{AMB}=$ atropina + midazolam + buprenorfina; $\mathrm{C}=$ clorpromazina; $\mathrm{M}=$ midazolam. $\mathrm{MPA}=$ medicação pré-anestésica; $\mathrm{EPI}=$ epidural; $\mathrm{M} 0=$ controle; M3 = 15 minutos após a MPA; M5 = 30 minutos após a EPI.

Os protocolos ACB e M produziram aumento na freqüência cardíaca após a realização da epidural, enquanto que o protocolo AX foi responsável por marcada redução da freqüência cardíaca imediatamente após a medicação pré-anestésica.

Com relação à pressão arterial sistólica, todos os protocolos garantiram sua manutenção dentro dos limites observados na medida controle, exceto o protocolo $\mathrm{M}$, que acarretou elevação em todas as medidas, após a MPA.

Uma Redução da temperatura retal foi observada em todos os protocolos, sendo estatisticamente significativa nas aferições realizadas em M4 (15 minutos após a EPI), M5 (30 minutos após a EPI), M6 (45 minutos após a EPI) e M7 (60 minutos após a EPI).

Os resultados de $\mathrm{pH}$ sangüíneo, bicarbonato e excesso base estão resumidos na tabela 2. As flutuações observadas, diferindo entre protocolos, não mostraram diferenças para os valores prévios de cada grupo.

Os resultados de pressão parcial de $\mathrm{CO}_{2}$ sangüíneo, $\mathrm{CO}_{2}$ total, pressão parcial de $\mathrm{O}_{2}$ sangüíneo e saturação de oxihemoglobina estão apresentados na tabela 3. Como regra, as alterações de $\mathrm{P}_{\mathrm{v}} \mathrm{CO}_{2}$ sangüíneo foram no sentido da elevação, na comparação com as aferições prévias (MO). Entretanto, diferença estatisticamente significativa só foi observada no protocolo $\mathrm{AMB}$, na tomada realizada 30 minutos após a EPI.

Com relação à $\mathrm{P}_{\mathrm{v}} \mathrm{O}_{2}$ sangüíneo, não houve um padrão de redução em todos os protocolos.

A avaliação do ritmo cardíaco durante o 
Tabela 3. Pressão parcial de $\mathrm{CO}_{2}$ sangüíneo, $\mathrm{CO}_{2}$ total, pressão parcial de $\mathrm{O}_{2}$ sangüíneo e saturação de oxihemoglobina sangüínea (médias \pm desvio padrão) de cães $(n=5$ por grupo) submetidos a seis protocolos pré-anestésicos e então à epidural com lidocaína 2\% com adrenalina 1:200000 + bupivacaína $0,5 \%$ com adrenalina 1:200000 (1:1).

\begin{tabular}{|c|c|c|c|c|}
\hline $\begin{array}{l}\text { Tempos } \\
\text { Variável } \\
\end{array}$ & MPA & M0 & M3 & M5 \\
\hline \multirow{6}{*}{$\begin{array}{l}\text { Pressão } \\
\text { parcial venosa } \\
\text { de } \mathrm{CO}_{2} \\
(\mathrm{~mm} \text { de } \mathrm{Hg})\end{array}$} & $\mathrm{AX}$ & $51,8 \pm 3,1$ & $54,8 \pm 5,4^{b}$ & $53,8 \pm 7,3$ \\
\hline & $\mathrm{ACB}$ & $47,0 \pm 6,4$ & $45,8 \pm 4,0^{\mathrm{a}}$ & $53,6 \pm 9,1$ \\
\hline & $\mathrm{MC}$ & $46,0 \pm 7,1$ & $44,8 \pm 4,5^{\mathrm{a}}$ & $50,4 \pm 5,6$ \\
\hline & AMB & $47,8 \pm 3,7^{1}$ & $49,0 \pm 2,0^{\text {lab }}$ & $55,6 \pm 4,9^{2}$ \\
\hline & $\mathrm{C}$ & $49,0 \pm 5,2$ & $51,0 \pm 3,3^{\mathrm{ab}}$ & $53,0 \pm 3,6$ \\
\hline & M & $49,4 \pm 6,4$ & $49,6 \pm 2,9^{\mathrm{ab}}$ & $52,4 \pm 4,0$ \\
\hline \multirow{6}{*}{$\begin{array}{l}\mathrm{CO}_{2} \text { total } \\
(\mathrm{mmol} / \mathrm{l})\end{array}$} & $\mathrm{AX}$ & $24,0 \pm 3,3^{\mathrm{a}}$ & $24,0 \pm 2,7^{\mathrm{a}}$ & $23,0 \pm 3,0^{\mathrm{a}}$ \\
\hline & $\mathrm{ACB}$ & $28,0 \pm 2,3^{\mathrm{ab}}$ & $28,4 \pm 2,1^{b}$ & $28,6 \pm 2,3^{\mathrm{bc}}$ \\
\hline & $\mathrm{MC}$ & $23,8 \pm 2,3^{\mathrm{a}}$ & $23,0 \pm 2,3^{a}$ & $24,0 \pm 2,7^{\mathrm{ab}}$ \\
\hline & AMB & $29,4 \pm 1,5^{b}$ & $29,2 \pm 1,3^{b}$ & $29,8 \pm 1,6^{\mathrm{c}}$ \\
\hline & $\mathrm{C}$ & $30,2 \pm 1,7^{\mathrm{b}}$ & $30,0 \pm 1,8^{b}$ & $30,0 \pm 2,3^{c}$ \\
\hline & M & $27,2 \pm 2,5^{\mathrm{ab}}$ & $28,4 \pm 2,3^{b}$ & $28,0 \pm 2,9^{\mathrm{bc}}$ \\
\hline \multirow{7}{*}{$\begin{array}{l}\text { Pressão } \\
\text { parcial venosa } \\
\text { de } \mathrm{O}_{2} \\
(\mathrm{~mm} \text { de } \mathrm{Hg})\end{array}$} & $\mathrm{AX}$ & $36,4 \pm 4,5^{1}$ & $34,6 \pm 4,6^{1 a}$ & $51,4 \pm 4,6^{2 b}$ \\
\hline & $\mathrm{ACB}$ & $33,8 \pm 7,0^{1}$ & $43,2 \pm 3,4^{2 a b}$ & $32,4 \pm 5,0^{1 \mathrm{a}}$ \\
\hline & $\mathrm{MC}$ & $47,2 \pm 18,7$ & $46,8 \pm 4,0^{\mathrm{b}}$ & $38,612,4^{\mathrm{ab}}$ \\
\hline & AMB & $33,6 \pm 5,8$ & $38,0 \pm 4,1^{\mathrm{ab}}$ & $39,4 \pm 7,5^{\mathrm{ab}}$ \\
\hline & $\mathrm{C}$ & $38,8 \pm 6,2$ & $35,2 \pm 2,7^{\mathrm{a}}$ & $40,4 \pm 7,2^{\mathrm{ab}}$ \\
\hline & M & $36,4 \pm 3,2$ & $39,0 \pm 7,7^{\mathrm{ab}}$ & $42,8 \pm 6,8^{\mathrm{ab}}$ \\
\hline & $\mathrm{AX}$ & $59,6 \pm 6,8$ & $54,6 \pm 6,4^{\mathrm{a}}$ & $77,6 \pm 11,0$ \\
\hline \multirow{5}{*}{$\begin{array}{l}\text { Saturação de } \\
\text { Oxihemoglobina } \\
(\%)\end{array}$} & $\mathrm{ACB}$ & $60,8 \pm 13,2^{12}$ & $77,6 \pm 4,3^{2 \mathrm{c}}$ & $56,6 \pm 11,8^{1}$ \\
\hline & $\mathrm{MC}$ & $64,0 \pm 6,4$ & $77,8 \pm 5,2^{\mathrm{c}}$ & $60,4 \pm 19,6$ \\
\hline & AMB & $62,0 \pm 11,2$ & $70,0 \pm 5,5^{\mathrm{bc}}$ & $67,8 \pm 11,6$ \\
\hline & $\mathrm{C}$ & $70,8 \pm 8,7$ & $64,6 \pm 6,0^{\mathrm{ab}}$ & $71,2 \pm 8,6$ \\
\hline & M & $65,2 \pm 6,3$ & $68,6 \pm 10,4^{\mathrm{bc}}$ & $72,6 \pm 11,2$ \\
\hline
\end{tabular}

Para cada variável, letras diferentes na mesma coluna representam diferença estatisticamente significativa entre os protocolos, e números diferentes na mesma linha representam diferença estatisticamente significativa entre os momentos $(\mathrm{p}<0,05)$.

$\mathrm{AX}=$ atropina + xilazina; $\mathrm{ACB}=$ atropina + clorpromazina + buprenorfina; $\mathrm{MC}=$ midazolam + clorpromazina; $\mathrm{AMB}=$ atropina + midazolam + buprenorfina; $\mathrm{C}=$ clorpromazina; $\mathrm{M}=$ midazolam. $\mathrm{MPA}=$ medicação pré-anestésica; $\mathrm{EPI}=$ epidural; $\mathrm{M} 0$ = controle; M3 = 15 minutos após a MPA; M5 = 30 minutos após a EPI.

período experimental mostrou a ocorrência de diferentes alterações em diferentes momentos, e variáveis conforme o protocolo. Com o protocolo AX a maioria das alterações de ritmo ocorreram entre 10 minutos após a MPA (M2) e 15 minutos após a MPA (M3) e constaram de: arritmia sinusal, marcapasso ectópico, bloqueio atrioventricular de primeiro e segundo grau, taquicardia, bloqueio sinoatrial, batimento ventricular prematuro e dissociação atrioventricular.

Com o protocolo ACB prevaleceu a ocorrência de ritmo sinusal normal e arritmia sinusal durante todo o procedimento experimental. As alterações observadas foram marcapasso ectópico e bloqueio atrioventricular de segundo grau, 10 minutos após a MPA(M2) e taquicardia sinusal a partir dos 15 minutos após a MPA (M3), até o final do procedimento.

No protocolo MC prevaleceu a ocorrência de ritmo sinusal normal durante as avaliações realizadas. As alterações observadas contaram de marcapasso ectópico entre M1 (5 minutos após a MPA) e M5 (30 minutos após a EPI) e, taquicardia sinusal e batimentos ventriculares prematuros entre 15 e 30 minutos após a EPI (M4 e M5).

As alterações de ritmo mais marcantes acarretadas pelo protocolo AMB ocorreram entre M1 (5 minutos após a MPA) e M2 (10 minutos após a 
Tabela 4. Período de latência da medicação pré-anestésica (MPA) em minutos, tempo até o relaxamento do esfíncter anal (REA) em segundos e, período de latência da epidural (EPI) em minutos (valores médios \pm desvio padrão) de cães (5 animais por grupo) submetidos a 6 protocolos pré-anestésicos distintos e então a EPI com lidocaína 2\% com adrenalina 1:200000 + bupivacaína 0,5\% com adrenalina 1:200000 (1:1).

\begin{tabular}{lccc}
\hline $\begin{array}{c}\text { Variável } \\
\text { Protocolo MPA }\end{array}$ & $\begin{array}{c}\text { Latência da MPA } \\
\text { (minutos) }\end{array}$ & $\begin{array}{c}\text { Tempo até REA } \\
\text { (segundos) }\end{array}$ & $\begin{array}{c}\text { Latência da EPI } \\
\text { (minutos) }\end{array}$ \\
\hline AX & $8,6 \pm 2,4$ & $21,8 \pm 2,5$ & $5,6 \pm 3,2$ \\
ACB & $3,0 \pm 3,2$ & $22,0 \pm 2,9$ & $5,6 \pm 3,5$ \\
MC & $5,8 \pm 3,6$ & $21,2 \pm 2,1$ & $6,8 \pm 3,8$ \\
AMB & $7,8 \pm 2,5$ & $20,2 \pm 3,2$ & $5,8 \pm 3,3$ \\
C & $3,4 \pm 1,1$ & $23,0 \pm 1,5$ & $7,6 \pm 1,1$ \\
M & $14,6 \pm 0,5$ & $23,8 \pm 4,0$ & $8,2 \pm 1,3$ \\
\hline
\end{tabular}

$\mathrm{AX}=$ atropina + xilazina; $\mathrm{ACB}=$ atropina + clorpromazina + buprenorfina $; \mathrm{MC}=$ midazolam + clorpromazina $; \mathrm{AMB}=$ atropina + midazolam + buprenorfina; $\mathrm{C}=$ clorpromazina; $\mathrm{M}$ = midazolam.

MPA) e constaram de marcapasso ectópico, bloqueio atrioventricular de primeiro e segundo graus, taquicardia sinusal, batimento ventricular prematuro e arritmia ventricular.

Os ritmos prevalentes durante as aferições do protocolo $\mathrm{C}$ foram o sinusal normal e arritmia sinusal, porém, bloqueio sinusal esteve presente em todos os momentos investigados. Marcapasso ectópico foi observado em dois momento, M5 (30 minutos após a EPI) e M6 (45 minutos após a EPI).

Com o protocolo $\mathrm{M}$ ocorreu marcapaço ectópico entre M1 (5 minutos após a MPA) e M3 (15 minutos após a MPA) e taquicardia sinusal entre M4 (15 minutos após a EPI) e M7 (60 minutos após a EPI).

Os resultados de período de latência da medicação pré-anestésica, tempo até o relaxamento do esfíncter anal e, período de latência da epidural estão apresentados na tabela 4. Com o protocolo ACB ocorreu o menor período de latência da MPA (3,0 minutos) e o maior com o protocolo $\mathrm{M}$ (14,6 minutos).

\section{DISCUSSÃO}

O limite fisiológico de freqüência respiratória para a espécie canina é de 10 a 40 movimentos por minuto [17]. O único grupo a apresentar valores abaixo do limite fisiológico foi o do protocolo AX em M3, M4, M6 e M7, comportamento que está de acordo com o anteriormente descrito na literatura [11, 25]. Os demais protocolos não determinaram alteração na freqüência respiratória, o que também corrobora as observações de outros autores que avaliaram as drogas investigadas $[14,15]$.

$\mathrm{O}$ limite fisiológico de pressão parcial de $\mathrm{CO}_{2}$ de amostras sangüíneas venosas para a espécie canina é de 30 a 50 milímetros de mercúrio [16]. No momento controle, todos os protocolos, exceto AX, apresentaram valores de pressão parcial de $\mathrm{CO}_{2}$ dentro dos limites fisiológicos, indicando padrões respiratórios inalterados. Doses elevadas de xilazina podem resultar em diminuição da freqüência respiratória e, em alguns casos, redução da pressão parcial de $\mathrm{CO}_{2}$ [11]. Após a anestesia epidural, todos os protocolos apresentaram valores de pressão parcial de $\mathrm{CO}_{2}$ acima do limite fisiológico, o que pode indicar a presença de desempenho respiratório prejudicado, provavelmente em decorrência da manipulação/estimulação durante a epidural.

O limite fisiológico de pressão parcial de $\mathrm{O}_{2}$ de amostras sangüíneas venosas para a espécie canina é de 40 a 60 milímetros de mercúrio [3]. Todos os protocolos, exceto o $\mathrm{MC}$, apresentaram valores basais abaixo dos limites fisiológicos. O protocolo AX apresentou uma redução inicial da pressão parcial de $\mathrm{O}_{2}$, sofrendo posterior compensação. Este mecanismo compensatório já havia sido registrado quando foram investigados os efeitos da xilazina [11]. 
Embora amostras de sangue venoso não substituam as arteriais na análise do perfil hemogasométrico, já foi constatado que as amostras de sangue capilar podem substituir as arteriais para avaliação do equilíbrio ácido-básico e gasometria sangüínea em cães com descompensação da função pulmonar [24]. O uso de amostras venosas para a análise hemogasométrica, também é validado por outros autores [26].

A marcada redução da freqüência cardíaca imediatamente após a medicação pré-anestésica causada pelo protocolo $\mathrm{AX}$ foi de caracter temporário, e encontra explicação nos efeitos agonistas alfa 2 da xilazina $[8,25]$. O caracter temporário da redução encontra explicação, pelo menos parcialmente, devido aos efeitos da atropina [25]. O aumento na freqüência cardíaca após a realização da epidural com os protocolos $\mathrm{ACB}$ e M pode ser decorrente da estimulação envolvida com o procedimento, e com a redução dos efeitos farmacológicos no momento da epidural [5].

Os efeitos hipotensores dos fármacos usados na pré-anestesia de cães são amplamente comprovados $[8,19,25]$. Dos protocolos avaliados, somente o AX mostrou redução da pressão arterial sistólica, ocorrendo aos 10 minutos após a MPA. Os demais protocolos não alteraram significativamente os valores de pressão arterial sistólica. A técnica de aferição não invasiva da pressão arterial pelo métodos de Doppler pode ter contribuído com estes resultados, sendo a aferição invasiva responsável pela obtenção de resultados mais acurados [2]. A elevação da pressão arterial pelo protocolo $\mathrm{M}$ permanece com sua causa a ser investigada.

Os ritmos sinusal normal e a arritmia sinusal com marcapasso migratório são considerados fisiológicos para a espécie canina segundo a American Academy of Veterinary Cardiology [22]. A presença de anormalidades no ritmo ou condução do impulso elétrico é considerada uma arritmia. As arritmias são fenômenos importantes porque diminuem o débito cardíaco, podendo, em casos severos levar ao colapso cardíaco, com morte súbita. As principais causas de arritmias nos períodos trans e pós anestésicos são decorrentes da liberação de catecolaminas, hipóxia, hipoventilação, depressão cardiovascular, hipercapnia, hiper ou hipotermia, distúrbios eletrolíticos, hiper ou hipovolemia, insuficiência cardíaca congestiva, acidose ou alcalose, distúrbios gastrointestinais, traumatismos cranianos e torácicos, choque, toxemia e efeito de fármacos usados na anestesia [21]. As diferentes alterações do ritmo cardíaco observadas com os diferentes protocolos utilizados encontram explicação primariamente nos fármacos empregados $[4,8,22,25]$. Por outro lado, a ocorrência de caracter esporádico e reversível das arritmias observadas reduz sua significância clínica.

Os períodos de latência da MPA, tempo até o relaxamento do esfíncter anal após a realização da epidural e o de latência da epidural foram bons indicadores para o início dos procedimentos. Todavia, nenhum dos protocolos causou sedação adequada, em intensidade e duração, capaz de permitir a realização da anestesia epidural.

\section{CONCLUSÕES}

É possível escolher a combinação a ser usada na pré-anestesia para a anestesia epidural em cães, baseado nas condições clínicas e temperamento dos animais. Todavia, sugere-se uma ampliação das doses empregadas, ou mesmo suplementação das mesmas nos casos em que um tempo adicional for necessário, para que a anestesia epidural seja realizada adequadamente, com mínima reação do animal.

\section{NOTAS INFORMATIVAS}

${ }^{1}$ Sulfato de atropina - Geyer Medicamentos S.A. Porto Alegre, RS.

${ }^{2}$ Dopaser - Laboratórios Calier S.A. Barcelona Espanha.

${ }^{3}$ Clorpromazina - Cristália Produtos Químicos e Farmacêuticos Ltda. Itapira, SP.

${ }^{4}$ Temgesic - Schering Plough Indústria Química e Farmacêutica S.A. Rio de Janeiro, RJ.

5 Dormonid - Roche Produtos Químicos e Farmacêuticos S.A. São Paulo, SP.

${ }^{6}$ Anestésico Bravet - Laboratório Bravet Ltda. Rio de Janeiro, RJ.

7 Neocaína - Cristália Produtos Químicos e Farmacêuticos. Itapira, SP. 
${ }^{8}$ Eletrocardiógrafo modelo $01 \mathrm{k}$ - Toshiba

${ }^{9}$ Pocket Doppler Model 841-A - Pars Medical Eletronics, Inc. USA.

${ }^{10}$ BD Becton Dickinson - Canada Inc. Miss. Ontario.

${ }^{11} \mathrm{pH}$ Blood Gas Analyser Modelo 248 - Ciba Corning Diagnostic Limited. Halstead, England.

\section{REFERÊNCIAS}

1 Bednarski R.M. 1996. Anesthesia and immobilization of specific species - dogs and cats. In: Jones L. Veterinary anesthesia. 3rd edn. Baltimore: Williams \& Wilkins, pp.591-598.

2 Bichard S.J. \& Sherding R.G. 1998. Manual Saunders - Clínica de Pequenos Animais. São Paulo: Roca, 1591 p.

3 Bistner S.I. \& Ford R.B. 1997. Manual de procedimentos veterinários e tratamento de emergências. 6. ed. São Paulo: Roca, 914 p.

4 Bolton G.R. 1975. Handbook of canine eletrocardiography. Philadelphia: W.B. Saunders, 350 p.

5 Canto S.P. 2000. Avaliação de seis protocolos préanestésicos na anestesia epidural de caninos. $250 \mathrm{f}$. Porto Alegre, RS. Dissertação (Mestrado em Ciências Veterinárias) - Programa de Pós-Graduação em Ciências Veterinárias, Universidade Federal do Rio Grande do Sul.

6 Cox D.R. 1992. The collected works of John W. TukeyFactorial \& ANOVA: 1946 - 1962. Pacific Grove: Brooks/ Cole Publishing Co., 336p.

Dripps N., Lamont A.\& Eckenhoff J.E. 1961. The role of anesthesia in surgical mortality. Journal of the American Medical Association. 178:107-112.

8 Fantoni D.T. \& Cortopassi S.R.G. 1998. Anestesiando pacientes cardiopatas. Cães \& Gatos. 5: 6-10.

9 Grosenbaugh D.A. \& Muir W.W. 1998. Blood pressure monitoring. Veterinary Medicine. 2: 48-74.

10 Hall L.W. 1992. Princípios generales de la anestesia. In: Manual de anestesia de los pequeños animales. 3 ed. Zaragoza: Acribia, pp.1-8.

11 Haskins S.C., Patz J.D. \& Farver T.B. 1986. Xylazine and xylazine-ketamine in dogs. American Journal of Veterinary Research. 47: 633-641.

12 Kelly W.R. 1986. Diagnóstico clínico veterinário. 3. ed. Rio de Janeiro: Panamericana, 364p.

13 Kirk R.W. \& Bistner S.I. 1987. Manual de procedimentos e tratamento de emergência em medicina veterinária. 3. ed. São Paulo: Manole, 994p.
14 Klide A.M., Calderwood H.W. \& Soma L.R. 1975. Cardiopulmonary effects of xylazine in dogs. American Journal of Veterinary Research. 36: 931-935.

15 Lavoie J.P., Pascoe J.R. \& Kurpershock C.J. 1992. Effects of xylazine on ventilation in horses. American Journal of Veterinary Research. 53: 916-920.

16 Lopes S.T., Cunha C.M.S., BiondoA.W. \& Fan L.C. 1996. Patologia clínica veterinária. Santa Maria: UFSM, 166p.

17 Massone F. 1988. Anestesiologia Veterinária - Farmacologia e técnicas. Rio de Janeiro: Guanabara, 235 p.

18 Mc Kelvey D. \& Hollingshead K.W. 1994. Small animal anesthesia - Canine and feline practice. St. Louis: Mosby, 332p.

19 Mueller R.A. \& Lundberg D.B.A. 1997. Manual de interações medicamentosas em anestesia. 2. ed. Rio de Janeiro: Revinter, 406p.

20 Muir W.W., Hubbell J.A., Skarda R.T. \& Bednarski R.M. 2001. Manual de Anestesia Veterinária. 3. ed. Porto Alegre: Artes Médicas, 432p.

21 Pereira P.M., Selmi A.L., Notomi M.K., Almeida E.M.P. \& Nunes, N. 1997. Tratamento de arritmias nos períodos trans e pós anestésicos. Clínica Veterinária.11: 31-34.

22 Serpa M.F. 1998. Noções básicas de cardiologia e eletrocardiografia. In: Curso de noções básicas de cardiologia e eletrocardiografia. Porto Alegre, RS.

23 Skarda R.T. 1996. Local and regional anesthetic and analgesic techniques in dogs. In: Lumb \& Jones Veterinary Anesthesia. 3rd edn. Baltimore: Williams \& Wilkins, pp. 426-447.

24 Sluijs F.J., Vries H.W., Bruijne J.J. \& Brom W.E. 1983. Capillary and venous blood compared with arterial blood in the measurement of acid-base and blood gas status of dogs. American Journal of Veterinary Research. 44: 459-462.

25 Thurmon J.C., Hurmon J.C., Tranquilli W.J. \& Benson G.J. 1996. Lumb \& Jones Veterinary anesthesia. 3rd edn. Baltimore: Williams \& Wilkins, $928 \mathrm{p}$.

26 Williams L.L., Boudrieau R.J., Clark G., Court M.H., Dodman N.H., Rand W. \& Agarwal R. 1993. Evaluation of epidural morphine in dogs for pain relief after hindlimb orthopedic surgery. Veterinary Surgery. 22: 89-93.

Pub. 521 